\title{
Uniqueness of meromorphic functions with their reduced linear $c$-shift operators sharing two or more values or sets
}

\author{
Abhijit Banerjee $^{1^{*}}$ and Saikat Bhattacharyya ${ }^{2}$
}

\section{"Correspondence:}

abanerjee_kal@yahoo.co.in;

abanerjeekal@gmail.com

'Department of Mathematics,

University of Kalyani, West Bengal, India

Full list of author information is available at the end of the article

\begin{abstract}
In the paper, we introduce a new notion of reduced linear $c$-shift operator $L_{c}^{r} f$, and with the aid of this new operator, we study the uniqueness of meromorphic functions $f(z)$ and $L_{c}^{r} f$ sharing two or more values in the extended complex plane. The results obtained in the paper significantly improve a number of existing results. Further, using the notion of weighted sharing of sets, we deal the same problem. We exhibit a handful number of examples to justify certain statements relevant to the content of the paper. We are also able to determine the form of the function that coincides with its reduced linear c-shift operator. At the end of the paper, we pose an open question for future research.
\end{abstract}

MSC: $30 \mathrm{D} 35$

Keywords: Meromorphic functions; Uniqueness; Difference operator; Shared set; Weighted sharing

\section{Introduction and results}

At the outset, we assume that the readers are familiar with the standard notations and basic results of Nevanlinna's value distribution theory (see $[6,14])$. Let $f$ be a nonconstant meromorphic functions defined in the open complex plane $\mathbb{C}$.

We recall that $T(r, f)$ denotes the Nevanlinna characteristic function of a nonconstant meromorphic function $f$ and $N\left(r, \frac{1}{f-a}\right)=N(r ; a ; f)\left(\bar{N}\left(r, \frac{1}{f-a}\right)=\bar{N}(r ; a ; f)\right)$ denotes the counting function (reduced counting function) of $a$-points of a meromorphic function $f$.

We need the following definitions and notations.

Definition 1.1 For a nonconstant meromorphic function $f$ and $S \subset \mathbb{C} \cup\{\infty\}$, let

$$
E_{f}(S)=\bigcup_{a \in S}\{(z, p) \in \mathbb{C} \times \mathbb{N}: f(z)=a \text { with multiplicity } p\}
$$

and

$$
\bar{E}_{f}(S)=\bigcup_{a \in S}\{(z, 1) \in \mathbb{C} \times \mathbb{N}: f(z)=a\}
$$

Then we say that $f$ and $g$ share the set $S$ CM (IM) if $E_{f}(S)=E_{g}(S)\left(\bar{E}_{f}(S)=\bar{E}_{g}(S)\right)$.

(C) The Author(s) 2019. This article is distributed under the terms of the Creative Commons Attribution 4.0 International License (http://creativecommons.org/licenses/by/4.0/), which permits unrestricted use, distribution, and reproduction in any medium, provided you give appropriate credit to the original author(s) and the source, provide a link to the Creative Commons license, and indicate if changes were made. 
When $S$ is a singleton set, the definition coincides with the traditional definition of value sharing.

In 2001, Lahiri [8, 9] introduced the definition of weighted sharing, which plays a key role in uniqueness theory as far as relaxation of sharing is concerned. In terms of Definition 1.1, weighted sharing of sets can be expressed as follows.

Definition 1.2 ([9]) Let $p$ be a nonnegative integer or infinity. For $a \in \mathbb{C} \cup\{\infty\}$, we denote by $E_{p}(a ; f)$ the set of all $a$-points of $f$, where an $a$-point of multiplicity $m$ is counted $m$ times if $m \leq p$ and $p+1$ times if $m>p$. For $S \subset \mathbb{C} \cup\{\infty\}$, we define $E_{f}(S ; k)=\bigcup_{a \in S} E_{k}(a ; f)$, where $k$ is a nonnegative integer or infinity. If $E_{f}(S ; k)=E_{g}(S ; k)$, then we say that $f$ and $g$ share the set $S$ with weight $k$ and write it as $(S, k)$.

Clearly, $E_{f}(S)=E_{f}(S, \infty)$ and $\bar{E}_{f}(S)=E_{f}(S, 0)$.

Definition 1.3 ([8]) We denote by $N_{2}(r, a ; f)$ the sum $\bar{N}(r, a ; f)+\bar{N}(r, a ; f \mid \geq 2)$.

Let $c$ be a nonzero complex constant, and let $f(z)$ be a meromorphic function. The shift operator is denoted by $f(z+c)$. Also, we use the notations $\Delta_{c} f$ and $\Delta_{c}^{k} f$ to denote the difference and $k$ th-order difference operators of $f(z)$, which are defined respectively by

$$
\Delta_{c} f(z)=f(z+c)-f(z), \quad \Delta_{c}^{k} f(z)=\Delta_{c}\left(\Delta_{c}^{k-1} f(z)\right), \quad k \in \mathbb{N}, k \geq 2 .
$$

Carefully observing the definitions, we see that all the variants of difference operators are nothing but linear combinations of different shift operators. So generalizing $\Delta_{c}^{k} f$, it will be reasonable to introduce the linear $c$-shift operator $L_{c} f=L_{c}(f)(z)$ as follows:

$$
L_{c} f=L_{c}(f)(z)=\sum_{j=0}^{k} a_{j} f(z+j c),
$$

where $a_{j} \in \mathbb{C}$ for $j=1,2, \ldots, k$ with $a_{k} \neq 0$. For convenience, putting $a_{k}=b_{k}, a_{k-1}=$ $-b_{k-1}, \ldots, a_{0}=(-1)^{k} b_{0}$, where $b_{i}$ are nonzero complex constants with $\sum_{j=0}^{k}(-1)^{k-j} b_{j}=0$, we get a special operator denoted by $L_{c}^{r} f=L_{c}^{r}(f)(z)$ and call it the reduced linear $c$-shift operator.

Putting $b_{k}=\left(\begin{array}{c}k \\ k\end{array}\right), b_{k-1}=\left(\begin{array}{c}k \\ k-1\end{array}\right), b_{k-2}=\left(\begin{array}{c}k \\ k-2\end{array}\right), \ldots, b_{0}=\left(\begin{array}{c}k \\ 0\end{array}\right)$ in $L_{c}^{r} f$, we easily verify that $L_{c}^{r}(f)(z)=\Delta_{c}^{k} f$.

The problem of uniqueness of meromorphic functions sharing two values were first initiated by Rubel and Yang [12] as follows.

Theorem A ([12]) Let $f$ be a nonconstant entire function. If $f$ and $f^{\prime}$ share two distinct values $(a, \infty)$ and $(b, \infty)$, then $f \equiv f^{\prime}$.

In 1979, relaxing the nature of sharing, Mues and Steinmetz [11] improved Theorem A as follows.

Theorem B ([11]) Let $f$ be a nonconstant entire function. If $f$ and $f^{\prime}$ share two distinct values $(a, 0)$ and $(b, 0)$, then $f \equiv f^{\prime}$. 
As derivative and difference operators are two natural extensions of a function, there is an intimate relation between them. So it is natural to investigate an analogous result corresponding to Theorem $\mathrm{B}$ under $\Delta_{c} f$.

In this direction, some progress has been made by Zhang and Liao [15].

Theorem C ([15]) Let $f(z)$ be a transcendental entire function of finite order, $c$ be a nonzero constant, and $a, b$ be two distinct finite constants. If $\Delta_{c} f(\not \equiv)$ and $f$ share $(a, \infty)$ and $(b, \infty)$, then $f \equiv \Delta_{c} f$. Furthermore, $f(z)$ must be of the form $f(z)=2^{\frac{z}{c}} h(z)$, where $h(z)$ is an entire function with period $c$.

Considering $f(z)=e^{\pi i z}$, Zhang and Liao [15] showed that though $f(z)$ and $\Delta_{1} f$ share 0 $\mathrm{CM}$, Theorem $\mathrm{C}$ ceases to hold. From the next example we easily see that in Theorem $\mathrm{C}$, two value sharing cannot even be replaced by one "nonzero" value sharing.

Example 1.1 Let $f(z)=e^{\left(\frac{i \pi}{4}+\frac{1}{2} \log 2\right) \frac{z}{c}}+1+i$. Then $f$ and $\Delta_{c} f=i e^{\left(\frac{i \pi}{4}+\frac{1}{2} \log 2\right) \frac{z}{c}}$ share $1 \mathrm{CM}$. Here $\Delta_{c} f=\Delta_{c}^{4 n+1} f, n \in \mathbb{N}$.

In fact, we can easily form the following series of examples, rather to say counterexamples, which fortify the fact that in Theorem $C$, two value sharing cannot be replaced by one "nonzero" value sharing.

Example 1.2 Let $f(z)=e^{\left(\frac{7 i \pi}{4}+\frac{1}{2} \log 2\right) \frac{z}{c}}+1-i$. Then $f$ and $\Delta_{c} f=-i e^{\left(\frac{7 i \pi}{4}+\frac{1}{2} \log 2\right) \frac{z}{c}}$ share $1 \mathrm{CM}$, where $\Delta_{c} f=\Delta_{c}^{4 n+1} f, n \in \mathbb{N}$.

Example 1.3 Let $f(z)=e^{\frac{i \pi z}{3 c}}+\frac{3}{2}+\frac{\sqrt{3} i}{2}$. Then $f$ and $\Delta_{c} f=\left(-\frac{1}{2}+\frac{\sqrt{3} i}{2}\right) e^{\frac{i \pi z}{3 c}}$ share $1 \mathrm{CM}$, where $\Delta_{c} f=\Delta_{c}^{3 n+1} f, n \in \mathbb{N}$.

Example 1.4 Let $f(z)=e^{\frac{2 i \pi z}{3 c}}-\frac{1}{2}+\frac{3 \sqrt{3} i}{2}$. Then $f$ and $\Delta_{c} f=\left(-\frac{3}{2}+\frac{\sqrt{3} i}{2}\right) e^{\frac{2 i \pi z}{3 c}}$ share $\sqrt{3} i \mathrm{CM}$.

Example 1.5 Let $f(z)=e^{\frac{4 i \pi z}{3 c}}+\frac{1}{2}+\frac{3 \sqrt{3} i}{2}$. Then $f$ and $\Delta_{c} f=\left(-\frac{3}{2}-\frac{\sqrt{3} i}{2}\right) e^{\frac{4 i \pi z}{3 c}}$ share $\sqrt{3} i$ CM.

Example 1.6 Let $f(z)=e^{\frac{5 i \pi z}{3 c}}+\frac{3}{2}-\frac{\sqrt{3} i}{2}$. Then $f$ and $\Delta_{c} f=\left(-\frac{1}{2}-\frac{\sqrt{3} i}{2}\right) e^{\frac{5 i \pi z}{3 c}}$ share 1 CM, where $\Delta_{c} f=\Delta_{c}^{3 n+1} f, n \in \mathbb{N}$.

We note that in Theorems A, B, and C, researchers are engaged in finding the uniqueness of a function with its first difference operator, but all are practically tacit about higherorder difference operators.

Recently, for meromorphic functions, Jiang and Chen [7] obtained an analogous result corresponding to Theorem C.

Theorem D ([7]) Let $f(z)$ be a nonconstant meromorphic function of finite order such that $N(r, f)=S(r, f)$, let $c \in \mathbb{C}$ be a nonzero constant such that $f(z+c)-f(z) \not \equiv 0$, and let $a$ and $b$ be two nonzero distinct finite complex constants. Iff and $\Delta_{c} f$ share $(a, \infty)$ and $(b, \infty)$, then $f(z+c)=2 f(z)$.

The prime intention of this paper is to improve this theorem for the reduced linear $c$ shift operator. In fact, we have further relaxed the condition over poles in Theorem D. First, we state the following theorem, which improves Theorems $C$ and D. 
Theorem 1.1 Let $f$ be a nonconstant meromorphic function of finite order, let $c$ be a constant such that $f(z+c)-f(z) \neq \equiv$, and let $a, b$ be two distinct nonzero finite constants. Suppose $L_{c}^{r} f$ and $f(z)$ share $(a, \infty)$ and $(b, \infty)$. If $(k+1) N(r, f)+2(k+2) \bar{N}(r, f)<T(r, f)$, then $f \equiv L_{c}^{r} f$.

Earlier in 2013, Chen and Yi [3] took into account the sharing of infinity in the direction of Theorem D. Their result is as follows.

Theorem E ([3]) Let $f(z)$ be a transcendental meromorphic function such that its order of growth $\sigma(f)$ is not an integer or infinite, and let $c \in \mathbb{C}$ be a constant such that $f(z+c) \not \equiv f(z)$. If $\Delta_{c} f(z)$ and $f(z)$ share three distinct values $(a, \infty)$ and $(b, \infty),(\infty, \infty)$, then $f(z+c) \equiv$ $2 f(z)$.

Recently $\mathrm{Lu}$ and $\mathrm{Lu}[10]$ removed the restriction " $\sigma(f)$ is not an integer" in the theorem and proved the following result.

Theorem $\mathbf{F}([10])$ Let $f(z)$ be a transcendental meromorphic function of finite order, and let $c \in \mathbb{C}$ be a constant such that $f(z+c) \not \equiv f(z)$. If $\Delta_{c} f(z)$ and $f(z)$ share three distinct values $(a, \infty),(b, \infty)$, and $(\infty, \infty)$, then $f(z+c) \equiv 2 f(z)$.

Considering Theorems E and F, it will be pertinent to extend the theorems in the direction of Theorem 1.1. In this respect, in the following theorem, we see that at the expense of allowing the sharing of $\{\infty\}$ along with the two shared values in Theorem 1.1, we have been able to remove the inequality and withdrawn the restriction over the sharing values to be nonzero.

Theorem 1.2 Let $f$ be a nonconstant transcendental meromorphic function of finite order that is not of period $c$, and let $a$ and $b$ be two distinct finite constants. Suppose $L_{c}^{r} f$ and $f(z)$ share $(a, \infty),(b, \infty)$, and $(\infty, \infty)$. Then $f \equiv L_{c}^{r} f$.

The following examples satisfy Theorem 1.1 for entire functions when $L_{c}^{r} f=\Delta_{c}^{k} f$ and $L_{c}^{r} f \neq \Delta_{c}^{k} f$.

Example 1.7 Let $f(z)=e^{n z}$. We choose $c=\frac{1}{n} \log 2$. Then for any even integer $k$ and $L_{c}^{r} f=$ $\Delta_{c}^{k} f$, it is easy to verify that the condition of Theorem 1.1 is satisfied and $\Delta_{c}^{k} f=f$.

Example 1.8 Let $f(z)=\sin \left(\frac{\pi z}{c}\right) e^{\frac{z \log \omega}{c}}$, where $c$ is a constant, and $\omega^{k}=1$.

When $k$ is an odd integer, choosing $b_{i}=-1,0 \leq i \leq k$, we have $\sum_{j=0}^{k}(-1)^{k-j} b_{j}=0$ and $L_{c}^{r} f=\omega^{k} f(z)+\omega^{k-1} f(z)+\omega^{k-2} f(z)+\cdots+f(z)=f(z)$.

When $k$ is an even integer, choosing $b_{i}(1+\omega)+1=\left(b_{0}+b_{k}\right)(1+\omega), 2 \leq i \leq k-1$, and $b_{1}=b_{0}+b_{k}$, we have $\sum_{j=0}^{k}(-1)^{k-j} b_{j}=0$ and

$$
\begin{aligned}
L_{c}^{r} f & =b_{k} \omega^{k} f(z)+b_{k-1} \omega^{k-1} f(z)+\cdots+b_{0} f(z) \\
& =\left(b_{0}+b_{k}\right) f(z)+b_{k-1} \omega^{k-1} f(z)+b_{k-2} \omega^{k-2} f(z)+\cdots+b_{1} \omega f(z) \\
& =b_{1}(1+\omega) f(z)+\left(\omega^{k-1}+\omega^{k-2}+\cdots+\omega^{2}\right)\left[b_{1}-\frac{1}{(1+\omega)}\right] f(z)=f(z) .
\end{aligned}
$$


The following examples satisfy Theorem 1.1 for meromorphic functions when $L_{c}^{r} f=$ $\Delta_{c}^{k} f$ and $L_{c}^{r} f \neq \Delta_{c}^{k} f$.

Example 1.9 Let $f(z)=e^{\frac{z \log 2}{c}} \frac{e^{\frac{6(k+3) \pi z i}{c}}}{e^{\frac{2 \pi z i}{c}}-1}$, where $c$ is a constant. Then for any even integer $k$ and $L_{c}^{r} f=\Delta_{c}^{k} f$, it is easy to verify that the condition of Theorem 1.1 is satisfied and $\Delta_{c}^{k} f=f$.

Example 1.10 Let $f(z)=\frac{e^{\frac{3(k+3) \pi z i}{c}}}{e^{\frac{2 \pi z i}{c}}-1}$, where $c$ is a nonzero constant. Here $f(z+s c)=f(z)$ when $s$ is even, and $=-f(z)$, when $s$ is odd. Then for any even integer $k$, choosing $b_{1}=b_{0}+b_{k}$, $b_{2}=b_{3}=\cdots=b_{k-1}$, and $2 b_{1}+(k-2) b_{i}-1=0,2 \leq i \leq k-1$, we have $\sum_{j=0}^{k}(-1)^{k-j} b_{j}=0$ and $L_{c}^{r} f=f(z)$.

With the observation as made in Sect. 4, we can construct the following examples satisfying Theorem 1.2 for entire functions when $L_{c}^{r} f=\Delta_{c}^{k} f$ and $L_{c}^{r} f \neq \Delta_{c}^{k} f$.

Example 1.11 Let $f(z)=\left\{\alpha_{1}^{\frac{z}{c}}+\alpha_{2}^{\frac{z}{c}}+\cdots+\alpha_{k}^{\frac{z}{c}}\right\} \exp \left(\frac{2 z \pi i}{c}\right)$. Choosing $\alpha_{i}, 1 \leq i \leq k$, as the roots of the equation $(1-z)^{k}-1=0$, it is easy to verify that $\Delta_{c}^{k} f=f$.

Example 1.12 Let $f(z)=\left\{\alpha_{1}^{\frac{z}{c}}+\alpha_{2}^{\frac{z}{c}}+\cdots+\alpha_{k}^{\frac{z}{c}}\right\} \sin \left(\frac{2 z \pi i}{c}\right)$.

When $k$ is odd integer, $\alpha_{i}, 1 \leq i \leq k$, are the roots of the equation $z^{k}-2 z+1=0$ for choosing $b_{i}=\frac{1}{2}, 0 \leq i \leq k$.

When $k$ is even integer, $\alpha_{i}, 1 \leq i \leq k$, are the roots of the equation $z^{k}-(3-k) z+k-2=0$ for choosing $b_{0}=-\frac{k}{2}, b_{i}=-\frac{1}{2}$ for odd $i$, and $b_{i}=\frac{1}{2}$ for even $i$.

Clearly, in both the cases we have $\sum_{j=0}^{k}(-1)^{k-j} b_{j}=0$ and $L_{c}^{r} f=f(z)$.

In the same way, with the observation as made in Sect. 4, for a meromorphic function, we can construct the following examples satisfying Theorem 1.2 when $L_{c}^{r} f=\Delta_{c}^{k} f$ and $L_{c}^{r} f \neq \Delta_{c}^{k} f$.

Example 1.13 Let $f(z)=\left\{\alpha_{1}^{\frac{z}{c}}+\alpha_{2}^{\frac{z}{c}}+\cdots+\alpha_{k}^{\frac{z}{c}}\right\} \frac{1}{\sin \left(\frac{2 z \pi i}{c}\right)}$. Here for $L_{c}^{r} f=\Delta_{c}^{k} f$ and $\alpha_{i}, 1 \leq i \leq k$, being the roots of the equation $(1-z)^{k}-1=0$, it is easy to verify that $\Delta_{c}^{k} f=f$.

Remark 1.1 We note that for a suitable choice of $k, \alpha_{i}$ can be determined in Example 1.11 and 1.13 as $\alpha_{1}=0$ for $k=1, \alpha_{1}=0$ and $\alpha_{2}=2$ for $k=2, \alpha_{1}=0, \alpha_{2}=\frac{3+3 i}{2}$, and $\alpha_{2}=\frac{3-3 i}{2}$ for $k=3$, and so on. In fact, instead of the functions chosen in Examples 1.11 and 1.13, any periodic function of period $c$ can be selected.

Example 1.14 Let $f(z)=\left\{\alpha_{1}^{\frac{z}{\bar{c}}}+\alpha_{2}^{\frac{z}{c}}\right\} \frac{e^{\frac{6(k+3) \pi z i}{c}}}{e^{\frac{2 \pi z i}{c}}-1}$, where $c$ is a nonzero constant.

When $k$ is an odd integer, $\alpha_{i}, i=1,2$, are the roots of the equation $z^{2}-2 z-1=0$ for choosing $b_{0}=\frac{1}{2}, b_{1}=1, b_{2}=\frac{1}{2}$, and $b_{i}=0$ for $3 \leq i \leq k$.

When $k$ is an even integer, $\alpha_{i}, i=1,2$, are the roots of the equation $z^{2}+z=0$ for choosing $b_{0}=1, b_{1}=\frac{1}{2}, b_{2}=-\frac{1}{2}$, and $b_{i}=0$ for $3 \leq i \leq k$.

Clearly, in both the cases, we have $\sum_{j=0}^{k}(-1)^{k-j} b_{j}=0$ and $L_{c}^{r} f=f(z)$.

In the next example, we see that Theorem 1.1 is not valid when $f$ and $\Delta_{c}^{k} f$ share only one finite complex number. 
Example 1.15 Let $f(z)=\frac{A e^{d z / c}}{P(t)}+B$, where $A, B, d$, and $c$ are nonzero constants, and let $P(t)$ is a polynomial in $t=\sin ^{2}\left(\frac{\pi z}{c}\right)$ or $\cos ^{2}\left(\frac{\pi z}{c}\right)$. Then for each $k$, we have $\Delta_{c}^{k} f=\frac{A e^{d z / c}\left(e^{d}-1\right)^{k}}{P(t)}$. Now for $\alpha=\frac{B\left(e^{d}-1\right)^{k}}{\left(e^{d}-1\right)^{k}-1}$, it is easy to see that $f$ and $\Delta_{c}^{k} f$ share $\alpha$, but $f \not \equiv \Delta_{c}^{k} f$.

The following example shows that in Theorems 1.1 and 1.2 the relation $f \equiv \Delta_{c} f$ does not hold for a function $f$ of infinite order.

Example 1.16 Let $f(z)=e^{\phi(z)}+A$, where $\phi(z)=e^{\frac{z}{c} \log 2}$, and $c, A$ are nonzero constants. Then $\Delta_{c} f(z)=e^{2 \phi(z)}-e^{\phi(z)}$. Here we see that $f$ and $\Delta_{c} f(z)$ share two nonzero values $(A+$ $1)+\sqrt{A+1}$ and $(A+1)-\sqrt{A+1}$ but $f \not \equiv \Delta_{c} f$.

The next counterexample is an extension of this example for any $\Delta_{c}^{k} f$.

Example 1.17 Let $f(z)=e^{\sin \left(\frac{\pi z}{c}\right)}$, where $c$ is a nonzero constant. Then

$$
\Delta_{c}^{k} f(z)=(-1)^{k-1} 2^{k-1}\left(\frac{1-e^{2 \sin \left(\frac{\pi z}{c}\right)}}{e^{\sin \left(\frac{\pi z}{c}\right)}}\right) .
$$

Here we see that $f$ and $\Delta_{c}^{k} f$ share two nonzero values $\pm \sqrt{\frac{(-1)^{k-1} 2^{k-1}}{1+(-1)^{k-1} 2^{k-1}}} \mathrm{CM}$, but $f \not \Delta_{c}^{k} f$.

In view of Theorems 1.1 and 1.2, it is interesting to investigate the conditions under which the conclusions of Theorems 1.1 and 1.2 hold when sharing of the values $a$ and $b$ are relaxed from CM to IM. In our next two theorems, we deal in this regard.

Theorem 1.3 Let $f$ be a nonconstant meromorphic function of finite order, let $c$ be a constant such that $f(z+c)-f(z) \not \equiv 0$, and let $a$ and $b$ be two nonzero distinct finite constants. Suppose $L_{c}^{r} f$ and $f(z)$ share $(a, 0),(b, 0)$. If $3(k+1) N(r, f)+(k+4) \bar{N}(r, f)+3 \bar{N}\left(r, \frac{1}{f}\right)<T(r, f)$, then $f \equiv L_{c}^{r} f$.

Theorem 1.4 Let $f$ be a nonconstant meromorphic function of finite order, let $c$ be a constant such that $f(z+c)-f(z) \not \equiv 0$, and let $a$ and $b$ be two nonzero distinct finite constants. Suppose $L_{c}^{r} f$ and $f(z)$ share $(a, 0),(b, 0)$, and $(\infty, \infty)$. If $(k+1) N(r, f)+(k+4) \bar{N}(r, f)+$ $3 \bar{N}\left(r, \frac{1}{f}\right)<T(r, f)$, then $f \equiv L_{c}^{r} f$.

The following example shows that in Theorem 1.1 or Theorem 1.2, two finite value sharing cannot be replaced by sharing a set containing two elements for $L_{c}^{r} f=\Delta_{c}^{k} f$.

Example 1.18 Let $f(z)=\lambda \frac{\exp \left\{\frac{z}{l} \log (1+\lambda)\right\}}{\sin \left(\frac{2 \pi z}{c}\right)}$, where $c, \lambda$ are constants such that $\lambda^{k+1}=-1$. Then

$$
\Delta_{c}^{k} f(z)=\lambda^{k+1} \frac{\exp \left\{\frac{z}{c} \log (1+\lambda)\right\}}{\sin \left(\frac{2 \pi z}{c}\right)}
$$

Clearly, $f$ and $\Delta_{c}^{k} f$ share the set $\left\{\omega^{p}, \omega^{p+2^{n-1}}\right\}$, where $\omega=e^{\frac{2 \pi i}{2^{n}}}$, and $n$ and $p$ are two integers such that g.c.d. $\left(p, 2^{n}\right)=1$. Here $f \not \equiv \Delta_{c}^{k} f$.

So it is also interesting to see whether the two value sharing results concerning the uniqueness of $f$ and $\Delta_{c}^{k} f$ can be extended up to two set sharing. In this respect, we have the next two theorems, where the functions $f$ and $\Delta_{c}^{k} f$ share two sets. 
Theorem 1.5 Let $S_{1}=\left\{a w^{i-1}(z) \mid i=1,2, \ldots, n\right\}$ and $S_{2}=\left\{b u^{i-1}(z) \mid i=1,2, \ldots, m\right\}$, where $w=$ $\exp \left(\frac{2 \pi}{n}\right)$ and $u=\exp \left(\frac{2 \pi}{m}\right)$, and $a$ and $b$ are two constants satisfying $a b \neq 0$ and $a^{2 m n} \neq b^{2 m n}$. Suppose $f(z)$ is a nonconstant meromorphic function of finite order satisfying $E_{f}\left(S_{i}, 2\right)=$ $E_{\Delta_{c}^{k} f}\left(S_{i}, 2\right)$ for $i=1,2$, where $n \geq 9$ and $m \geq 9$ have no common factors. Then $f \equiv \Delta_{c}^{k} f$.

Theorem 1.6 Let $S_{i}$ for $i=1,2$ be defined as in Theorem 1.5. Suppose $f(z)$ is a nonconstant entire function of finite order such that $E_{f}\left(S_{i}, 1\right)=E_{\Delta_{c}^{k} f}\left(S_{i}, 1\right)$ for $i=1,2$, where $n \geq 5$ and $m \geq 5$ have no common factors. Then $f \equiv \Delta_{c}^{k} f$.

The following examples show that Theorems 1.5 and 1.6 are not valid if the two functions $f$ and $\Delta_{c}^{k} f$ share only one set.

Example 1.19 Let $f(z)=\phi(z)$, where $\phi(z)=\frac{\exp \left\{\frac{z}{\left.\frac{z}{\log }(1+\lambda)\right\}}\right.}{\sin \left(\frac{2 \pi z}{c}\right)}$, where $c$ is a constant, and for $\zeta=\cos \frac{2 \pi}{n}+i \sin \frac{2 \pi}{n}, \lambda$ is a root of the equation $\zeta^{n-1} z^{2 s}-\left(\zeta^{2 n-2}+1\right) z^{s}+\zeta^{n-1}=0$. Then clearly, $\Delta_{c}^{s} f(z)=\zeta^{n-1} \phi(z)$ or $\zeta^{1-n} \phi(z)$. Therefore for a nonzero constant $\alpha, f$ and $\Delta_{c}^{k} f$ share the set $\left\{\alpha, \alpha \zeta, \alpha \zeta^{2}, \ldots, \alpha \zeta^{n-1}\right\}$, but $f \not \equiv \Delta_{c}^{k} f$.

Example 1.20 Let $f(z)=\phi(z)$, where $\phi(z)=\exp \left\{\frac{z}{c} \log (1+\lambda)\right\}$, where $c$ and $\lambda$ are defined as in the previous example. Then clearly, $\Delta_{c}^{s} f(z)=\zeta^{n-1} \phi(z)$. Therefore for a nonzero constant $\alpha, f$ and $\Delta_{c}^{k} f$ share the set $\left\{\alpha, \alpha \zeta, \alpha \zeta^{2}, \ldots, \alpha \zeta^{n-1}\right\}$, but $f \not \equiv \Delta_{c}^{k} f$.

Note 1.1 In the previous examples, $f$ and $\Delta_{c}^{k} f$ share the set $\left\{\beta, \beta \zeta, \beta \zeta^{2}, \ldots, \beta \zeta^{k-1}\right\}$, where $\beta \neq \alpha$ is a constant, but $f \not \equiv \Delta_{c}^{k} f$. Note that here $m$ and $n$ have common factors.

Based on Theorems 1.5 and 1.6, it is relevant to investigate whether analogous results can be established by replacing two shared set problem with one shared set together with one shared value. In this regard, we have the following results.

Theorem 1.7 Let $S_{1}$ be the set defined as in Theorem 1.5, and let $S_{2}=\{b\}$, where $a \neq 0$, $b \neq 0$, and $b^{2 n} \neq a^{2 n}$. Suppose $f(z)$ is a nonconstant entire function of finite order such that $E_{f}\left(S_{i}, 1\right)=E_{\Delta_{c}^{k} f}\left(S_{i}, 1\right)$ for $i=1,2$ where $n \geq 5$. Then $f \equiv \Delta_{c}^{k} f$.

However, in the following theorem, we will show that to replace an entire function by a meromorphic one, the set $S_{2}$ must contain two elements.

Theorem 1.8 Let $S_{1}$ be the set defined as in Theorem 1.5, and let $S_{2}=\left\{b_{1}, b_{2}\right\}$, where $b_{1}$ and $b_{2}$ are two distinct nonzero constants such that $b_{1}^{n} \neq b_{2}^{n}, b_{i}^{2 n} \neq a^{2 n}$ for $i=1,2$, and $b_{1}^{n} b_{2}^{n} \neq a^{2 n}$. Suppose $f(z)$ is a nonconstant meromorphic function of finite order such that $E_{f}\left(S_{i}, 2\right)=$ $E_{\Delta_{c}^{k} f}\left(S_{i}, 2\right)$ for $i=1,2$ where $n \geq 9$. Then $f \equiv \Delta_{c}^{k} f$.

As $\Delta_{c} f$ and $\Delta_{c}^{k} f$ are nothing but the derivations from the shift operator $f(z+c)$ of the function $f$, it is interesting to explore whether analogous result corresponding to Theorem 1.1 can be obtained when $f(z)$ and $f(z+c)$ share a set with two elements.

The following examples show that like for the difference operator, it is again not possible for meromorphic and entire functions.

Example 1.21 Let $f(z)=\frac{2 e^{\frac{\pi i z}{c}}}{1+e^{\frac{2 \pi i z}{c}}}$, where $c$ is a constant. Then we see that for a nonzero constant $\alpha, f(z)$ and $f(z+c)$ share the set $\{\alpha,-\alpha\}$, but $f(z) \not \equiv f(z+c)$. 
Example 1.22 Let $f(z)=\frac{1+e^{\frac{\pi i z}{c}}}{1-e^{\frac{\pi i z}{c}}}$, where $c$ is a constant. Then for a nonzero constant $\alpha, f(z)$ and $f(z+c)$ share the set $\left\{\alpha, \frac{1}{\alpha}\right\}$, but $f(z) \not \equiv f(z+c)$.

Example 1.23 Let $f(z)=\sin \left(\frac{\pi z}{2 c}\right)$, where $c$ is a constant. Clearly, $f(z)$ and $f(z+c)$ share the set $\left\{\frac{1}{\sqrt{2}},-\frac{1}{\sqrt{2}}\right\}$, but $f(z) \not \equiv f(z+c)$.

So we have the following results analogous to Theorems 1.7-1.8.

Theorem 1.9 Under the same situation as in Theorem 1.7, if $\Delta_{c}^{k} f$ is replaced by $f(z+c)$, then $f(z) \equiv f(z+c)$.

Theorem 1.10 Under the same situation as in Theorem 1.8, if $\Delta_{c}^{k} f$ is replaced by $f(z+c)$, then $f(z) \equiv f(z+c)$.

\section{Lemmas}

In this section, we present some lemmas, which will be needed to proceed further.

Lemma 2.1 ([14]) Let $f(z)$ be a nonconstant meromorphic function, and let $d_{j}(j=1, \ldots, q)$ be $q$ distinct complex numbers. Then we have

$$
m\left(r, \sum_{j=1}^{q} \frac{1}{f-d_{j}}\right)=\sum_{j=1}^{q} m\left(r, \frac{1}{f-d_{j}}\right)+O(1) .
$$

Lemma 2.2 $([4,5])$ Let $f$ be a meromorphic function of finite order, and let $\eta$ be a nonzero complex constant. Then

$$
m\left(r, \frac{f(z+\eta)}{f(z)}\right)+m\left(r, \frac{f(z)}{f(z+\eta)}\right)=S(r, f) .
$$

Lemma 2.3 ([5]) Let $f(z)$ be a meromorphic function of finite order, and let $c \neq 0$ be fixed. Then

$$
\begin{aligned}
& \bar{N}(r, f(z+c)) \leq \bar{N}(r, f(z))+S(r, f), \\
& N(r, f(z+c)) \leq N(r, f(z))+S(r, f) .
\end{aligned}
$$

Lemma 2.4 ([7]) Let $f(z)$ be a nonconstant meromorphic function in $\mathbb{C}$. Let $d_{1}, d_{2}, \ldots, d_{n}$ be $n \geq 1$ distinct complex numbers. Then we have

$$
\begin{aligned}
& \sum_{j=1}^{n} m\left(r, \frac{1}{f-d_{j}}\right) \leq m\left(r, \frac{1}{f^{\prime}}\right)+S(r, f), \\
& \sum_{j=1}^{n} m\left(r, \frac{1}{f-d_{j}}\right) \leq m\left(r, \frac{1}{\Delta_{c} f}\right)+S(r, f) .
\end{aligned}
$$

Lemma 2.5 Let $f(z)$ be a nonconstant meromorphic function in $\mathbb{C}$. Let $d_{1}, d_{2}, \ldots, d_{n}$ be $n \geq 1$ distinct complex numbers. Then we have

$$
\sum_{j=1}^{n} m\left(r, \frac{1}{f-d_{j}}\right) \leq m\left(r, \frac{1}{L_{c}^{r} f}\right)+S(r, f) .
$$


Proof From the definition of $L_{c}^{r} f$ we have

$$
\begin{aligned}
L_{c}^{r} f= & \sum_{s=0}^{k}(-1)^{k-s} b_{s} f(z+s c) \\
= & b_{k}[f(z+k c)-f(z+\overline{k-1} c)]-\left(b_{k-1}-b_{k}\right)[f(z+\overline{k-1} c)-f(z+\overline{k-2} c)] \\
& +\left(b_{k-2}-b_{k-1}+b_{k}\right)[f(z+\overline{k-2} c)-f(z+\overline{k-3} c)]-\cdots+(-1)^{k-1} \\
& \times\left(b_{1}-b_{2}+b_{3}-\cdots+(-1)^{k-1} b_{k}\right)[f(z+c)-f(z)] .
\end{aligned}
$$

By Lemmas 2.1 and 2.2 we have

$$
\begin{aligned}
\sum_{j=1}^{n} m\left(r, \frac{1}{f-d_{j}}\right) & =m\left(r, \sum_{j=1}^{n} \frac{1}{f-d_{j}}\right)+O(1) \\
& =m\left(r, \frac{1}{L_{c}^{r} f} \cdot \sum_{j=1}^{n} \frac{L_{c}^{r} f}{f-d_{j}}\right)+O(1) \\
& \leq m\left(r, \frac{1}{L_{c}^{r} f}\right)+m\left(r, \sum_{j=1}^{n} \frac{L_{c}^{r} f}{f-d_{j}}\right)+O(1) \\
& =m\left(r, \frac{1}{L_{c}^{r} f}\right)+\sum_{j=1}^{n} m\left(r, \frac{L_{c}^{r} f}{f-d_{j}}\right)+O(1) \\
& \leq m\left(r, \frac{1}{L_{c}^{r} f}\right)+S(r, f) .
\end{aligned}
$$

Lemma 2.6 ([4]) Let $f(z)$ be a meromorphic function offinite order $\rho$, and let c be a nonzero complex constant. Then, for each $\epsilon>0$. we have

$$
T(r, f(z+c))=T(r, f(z))+O\left(r^{\rho-1+\epsilon}\right)+O(\log r) .
$$

Lemma 2.7 Let $f(z)$ be a meromorphic function of finite order, and let $c \in \mathbb{C} \backslash\{0\}$ be fixed. Then $S\left(r, L_{c}^{r} f\right)$ can be replaced by $S(r, f)$.

Proof In view of Lemmas 2.3 and 2.6, we have

$$
\begin{aligned}
T\left(r, L_{c}^{r} f\right) & =m\left(r, L_{c}^{r} f\right)+N\left(r, L_{c}^{r} f\right) \\
& \leq m\left(r, \frac{L_{c}^{r} f}{f}\right)+m(r, f)+N\left(r, L_{c}^{r} f\right)+o(T(r, f)) \leq(k+1+o(1)) T(r, f),
\end{aligned}
$$

and the lemma follows.

Lemma 2.8 ([13]) Let $F$ and $G$ be two nonconstant meromorphic functions such that $F$ and $G$ share the values $(1, \infty)$ and $(\infty, \infty)$. If

$$
\limsup _{r \rightarrow \infty} \frac{N_{2}(r, 0 ; F)+N_{2}(r, 0 ; G)+2 \bar{N}(r, \infty ; F)}{T(r)}<1, \quad r \in I,
$$

where $T(r)=\max \{T(r, F), T(r, G)\}$, then $F \equiv G$ or $F . G \equiv 1$. 
Lemma 2.9 ([2]) Let $F$ and $G$ share $(1,2)$. Then one of the following cases occurs:

(i) $\quad T(r, F) \leq N_{2}(r, 0 ; F)+N_{2}(r, 0 ; G)+N_{2}(r, \infty ; F)+N_{2}(r, \infty ; G)$ $+S(r, F)+S(r, G), \quad$ the same inequality holds for $T(r, G)$;

(ii) $F \equiv G$;

(iii) $F . G \equiv 1$.

Lemma 2.10 ([1]) Let $F$ and $G$ be nonconstant meromorphic functions defined in $\mathbb{C}$ such that they share $(1,1)$, and let

$$
H=\left(\frac{F^{\prime \prime}}{F^{\prime}}-\frac{2 F^{\prime}}{F-1}\right)-\left(\frac{G^{\prime \prime}}{G^{\prime}}-\frac{2 G^{\prime}}{G-1}\right) \not \equiv 0 .
$$

Then

$$
\begin{aligned}
T(r, F) \leq & N_{2}(r, 0 ; F)+N_{2}(r, \infty ; F)+N_{2}(r, 0 ; G)+N_{2}(r, \infty ; G) \\
& +\frac{1}{2} \bar{N}(r, 0 ; F)+\frac{1}{2} \bar{N}(r, \infty ; F)+S(r, F)+S(r, G) .
\end{aligned}
$$

\section{Proofs of theorems}

Proof of Theorem 1.1 Assume that $f \not \equiv L_{c}^{r} f$.

Using Lemma 2.7, we have

$$
\begin{aligned}
\bar{N} & \left(r, \frac{1}{L_{c}^{r} f-a}\right)+\bar{N}\left(r, \frac{1}{L_{c}^{r} f-b}\right) \\
& \leq N\left(r, \frac{1}{f-a}\right)+N\left(r, \frac{1}{f-b}\right) \\
& \leq N\left(r, \frac{1}{L_{c}^{r} f-f}\right) \\
& \leq T\left(r, L_{c}^{r} f-f\right)+S(r, f) \\
& =m\left(r, L_{c}^{r} f-f\right)+N\left(r, L_{c}^{r} f-f\right)+S(r, f) \\
& =m\left(r, f\left(\frac{L_{c}^{r} f}{f}-1\right)\right)+N\left(r, L_{c}^{r} f-f\right)+S(r, f) \\
& \leq m(r, f)+m\left(r, \frac{L_{c}^{r} f}{f}-1\right)+N\left(r, L_{c}^{r} f-f\right)+S(r, f) \\
& \leq m(r, f)+N\left(r, L_{c}^{r} f-f\right)+S(r, f) .
\end{aligned}
$$

From (3.1), in view of Lemma 2.3, we have

$$
\begin{gathered}
\bar{N}\left(r, \frac{1}{L_{c}^{r} f-a}\right)+\bar{N}\left(r, \frac{1}{L_{c}^{r} f-b}\right) \\
\quad \leq N\left(r, \frac{1}{f-a}\right)+N\left(r, \frac{1}{f-b}\right)
\end{gathered}
$$




$$
\begin{aligned}
& \leq T(r, f)+N\left(r, L_{c}^{r} f\right)+S(r, f) \\
& \leq T(r, f)+(k+1) N(r, f)+S(r, f) .
\end{aligned}
$$

Let

$$
U=\frac{\left(L_{c}^{r} f\right)^{\prime}}{L_{c}^{r} f-a}-\frac{f^{\prime}}{f-a} .
$$

Case 1. Suppose $U \not \equiv 0$.

As $L_{c}^{r} f$ and $f(z)$ share $(a, \infty)$, in view of Lemmas 2.3 and 2.7, a simple calculation yields

$$
\begin{aligned}
N(r, \infty ; U) & \leq \bar{N}\left(r, \infty ; L_{c}^{r} f\right)+\bar{N}(r, \infty ; f)+\bar{N}_{*}\left(r, a ; L_{c}^{r} f, f\right)+S(r, f) \\
& \leq(k+2) \bar{N}(r, f)+S(r, f) .
\end{aligned}
$$

Again using the logarithmic derivative theorem and Lemma 2.7, we get

$$
m(r, U) \leq S\left(r, L_{c}^{r} f\right)+S(r, f)=S(r, f) .
$$

Note that

$$
\frac{U}{f-b}=\frac{\left(L_{c}^{r} f\right)^{\prime}}{L_{c}^{r} f\left(L_{c}^{r} f-a\right)} \frac{L_{c}^{r} f}{f-b}-\frac{f^{\prime}}{(f-a)(f-b)} .
$$

So in view of the first fundamental theorem, (3.4), (3.5), and Lemmas 2.2 and 2.7, we get

$$
\begin{aligned}
& m\left(r, \frac{1}{f-b}\right) \\
&=m\left(r, \frac{1}{U}\right)+m\left(r, \frac{U}{f-b}\right) \\
& \leq T(r, U)+m\left(r, \frac{\left(L_{c}^{r} f\right)^{\prime}}{L_{c}^{r}\left(L_{c}^{r} f-a\right)}\right)+m\left(r, \frac{L_{c}^{r} f}{f-b}\right) \\
&+m\left(r, \frac{f^{\prime}}{(f-a)(f-b)}\right)+O(1) \\
& \leq T(r, U)+m\left(r, \frac{\left(L_{c}^{r} f\right)^{\prime}}{a}\left(\frac{1}{\left(L_{c}^{r} f-a\right)}-\frac{1}{L_{c}^{r} f}\right)\right)+m\left(r, \frac{L_{c}^{r} f}{f-b}\right) \\
&+m\left(r, \frac{f^{\prime}}{(a-b)}\left(\frac{1}{f-a)}-\frac{1}{(f-b)}\right)\right)+S(r, f) \\
& \leq(k+2) \bar{N}(r, f)+S\left(r, L_{c}^{r} f\right)+S(r, f) \leq(k+2) \bar{N}(r, f)+S(r, f) .
\end{aligned}
$$

In a similar manner, we can show that

$$
m\left(r, \frac{1}{f-a}\right) \leq(k+2) \bar{N}(r, f)+S(r, f) .
$$

Now from (3.2), (3.7), and (3.8), applying the first fundamental theorem, we obtain $2 T(r, f)+O(1)$ 


$$
\begin{aligned}
& =m\left(r, \frac{1}{f-a}\right)+m\left(r, \frac{1}{f-b}\right)+N\left(r, \frac{1}{f-a}\right)+N\left(r, \frac{1}{f-b}\right) \\
& \leq T(r, f)+(k+1) N(r, f)+2(k+2) \bar{N}(r, f)+S(r, f),
\end{aligned}
$$

which implies

$$
T(r, f) \leq(k+1) N(r, f)+2(k+2) \bar{N}(r, f)+S(r, f),
$$

a contradiction.

Case 2. Next, suppose $U \equiv 0$.

Integrating we get

$$
\left(L_{c}^{r} f-a\right)=C_{1}(f-a)
$$

where $C_{1}$ is a nonzero constant. In a similar way, we can get

$$
\left(L_{c}^{r} f-b\right)=C_{2}(f-b)
$$

where $C_{2}$ is a nonzero constant. If either of $C_{1}=1$ or $C_{2}=1$, then we are done. If $C_{1} \neq 1$ and $C_{2} \neq 1$, then from the last two equations, after simple calculations, we get

$$
\left(C_{1}-C_{2}\right) f(z)=C_{1} a-C_{2} b+b-a .
$$

If $C_{1} \neq C_{2}$, then $f$ is a constant, a contradiction. Therefore $C_{1}=C_{2}$, and hence $C_{1}(a-b)=$ $(a-b)$. As $a$ and $b$ are distinct, we have $C_{1}=C_{2}=1$, and so $f \equiv L_{c}^{r} f$.

Proof of Theorem 1.2 Suppose $f \not \equiv L_{c}^{r} f$.

Case $1 . a b \neq 0$.

Since $f$ and $L_{c}^{r} f$ share $(\infty, \infty)$, we have $N(r, f)=N\left(r, L_{c}^{r} f\right)$, and hence any pole of $L_{c}^{r} f$ or $f$ of multiplicity $p$ must be a pole of $L_{c}^{r} f-f$ of multiplicity $\leq p$, that is, $N\left(r, L_{c}^{r} f-f\right) \leq N(r, f)$.

So (3.1) reduces to

$$
\begin{aligned}
& \bar{N}\left(r, \frac{1}{L_{c}^{r} f-a}\right)+\bar{N}\left(r, \frac{1}{L_{c}^{r} f-b}\right) \\
& \quad \leq N\left(r, \frac{1}{f-a}\right)+N\left(r, \frac{1}{f-b}\right) \leq T(r, f)+S(r, f) .
\end{aligned}
$$

Let $U$ be defined as in (3.3).

Case 1.1 First, suppose $U \not \equiv 0$.

As $L_{c}^{r} f$ and $f(z)$ share $(a, \infty),(\infty, \infty)$, in view of Lemma 2.7 , we clearly have

$$
N(r, \infty ; U) \leq \bar{N}_{*}\left(r, \infty ; L_{c}^{r} f, f\right)+\bar{N}_{*}\left(r, a ; L_{c}^{r} f, f\right)=S(r, f) .
$$

Next, using (3.6), the first fundamental theorem, (3.5), (3.11), Lemmas 2.2 and 2.7 and proceeding in the same way as in Theorem 1.1, we see that (3.7) changes to

$$
m\left(r, \frac{1}{f-b}\right) \leq S\left(r, L_{c}^{r} f\right)+S(r, f)=S(r, f) .
$$


In a similar manner, we can show that

$$
m\left(r, \frac{1}{f-a}\right)=S(r, f)
$$

Now from (3.10), (3.12), and (3.13), applying the first fundamental theorem, similarly to (3.9), we obtain

$$
\begin{aligned}
& 2 T(r, f)+O(1) \\
& \quad=m\left(r, \frac{1}{f-a}\right)+m\left(r, \frac{1}{f-b}\right)+N\left(r, \frac{1}{f-a}\right)+N\left(r, \frac{1}{f-b}\right) \\
& \quad \leq T(r, f)+S(r, f),
\end{aligned}
$$

which implies

$$
T(r, f) \leq S(r, f)
$$

a contradiction.

Case 1.2. Next, suppose $U \equiv 0$.

Here we can prove the theorem in the same way as in Theorem 1.1. So we omit the details.

Case 2. Let $a b=0$.

Without loss of generality, we suppose that $b=0$. Let us consider the function

$$
\Phi=\frac{\left(L_{c}^{r} f\right)\left(L_{c}^{r} f-f\right)}{f(f-a)}
$$

\section{Case 2.1. First, suppose $\Phi \not \equiv 0$.}

Let $z_{0}$ be a zero of $f-a$ or that of $f$ of multiplicity $p$. As $L_{c}^{r} f$ and $f(z)$ share $(a, \infty)$ and $(0, \infty)$, clearly, $a$ - or 0 -points of $f$ will not be poles of $\Phi$. Noting that $f$ is a transcendental meromorphic function, each zero of $f$ of multiplicity $p$ will be a zero of $\Phi$ of multiplicity $\geq p$. Similarly, any pole of $f$ of multiplicity $q$ would be a pole of $L_{c}^{r} f-f$ of multiplicity $s \leq q$ and hence a pole of $\Phi$ of multiplicity $q+s-2 q \leq 0$. It follows that $\Phi$ has no pole. Also, from (3.14), Lemma 2.2, and the first fundamental theorem we have

$$
m(r, \Phi) \leq m\left(r, \frac{L_{c}^{r} f}{f-a}\right)+m\left(r,\left(\frac{L_{c}^{r} f}{f}-1\right)\right)+S(r, f) \leq S(r, f)
$$

So using the fact that $\Phi$ has no pole, from (3.15) we see that $S(r, \Phi)$ can be replaced by $S(r, f)$. Hence, in view of the first fundamental theorem, we have

$$
\begin{aligned}
N\left(r, \frac{1}{f}\right) & \leq N\left(r, \frac{1}{\Phi}\right)+S(r, f) \leq T\left(r, \frac{1}{\Phi}\right)-m\left(r, \frac{1}{\Phi}\right)+S(r, f) \\
& \leq T(r, \Phi)-m\left(r, \frac{1}{\Phi}\right)+S(r, f) \\
& \leq-m\left(r, \frac{1}{\Phi}\right)+S(r, f) .
\end{aligned}
$$


Again from Lemma 2.2 we note that

$$
\begin{aligned}
& m\left(r, \frac{1}{f}\right) \\
& \quad \leq m\left(r, \frac{1}{\Phi}\right)+m\left(r,\left(\frac{L_{c}^{r} f\left(L_{c}^{r} f-f\right)}{f^{2}(f-a)}\right)\right)+S(r, f) \\
& \quad \leq m\left(r, \frac{1}{\Phi}\right)+m\left(r, \frac{L_{c}^{r} f}{a}\left\{\frac{1}{f-a}-\frac{1}{f}\right\}\right)+m\left(r,\left(\frac{L_{c}^{r} f}{f}-1\right)\right)+S(r, f) \\
& \quad \leq m\left(r, \frac{1}{\Phi}\right)+S(r, f) .
\end{aligned}
$$

Using (3.17) in (3.16), from the first fundamental theorem we get

$$
T(r, f) \leq S(r, f)
$$

a contradiction.

Case 2.2. Next, suppose $\Phi \equiv 0$.

As we have $f \not \equiv L_{c}^{r} f$, clearly, $L_{c}^{r} f \equiv 0$, a contradiction. Hence the theorem follows.

Proof of Theorem 1.3 Assume that $f \not \equiv L_{c}^{r} f$.

For two complex constants $a$ and $b$ and two nonconstant meromorphic functions $f$ and $g$, by $N(r, a ; f \mid g=b)(N(r, a ; f \mid g \neq b))$ we mean the counting function of those $a$-points of $f$ that are (not) the $b$-points of $g$, where an $a$-point of $f$ is counted according to its multiplicity. Again for a positive integer $s$, by $N(r, a ; f \mid=s)$ we mean the reduced counting function of those $a$-points of $f$ that are of multiplicity exactly $s$.

As $L_{c}^{r} f$ and $f$ share $(a, 0)$ and $(b, 0)$, we see that an $a(b)$-point of $f$ whose multiplicity is greater than that of $L_{c}^{r} f$ is counted at least once in $N\left(r, \frac{1}{L_{c}^{r} f-f}\right)$. Thus

$$
\begin{aligned}
& \bar{N}\left(r, \frac{1}{L_{c}^{r} f-a}\right)+\bar{N}\left(r, \frac{1}{L_{c}^{r} f-b}\right) \\
& \leq N\left(r, \frac{1}{f-a}\right)+N\left(r, \frac{1}{f-b}\right) \\
& \quad \leq N\left(r, \frac{1}{L_{c}^{r} f-f}\right)+\{\bar{N}(r, a ; f \mid=2)+2 \bar{N}(r, a ; f \mid=3)+3 \bar{N}(r, a ; f \mid=4)+\cdots\} \\
& \quad+\{\bar{N}(r, b ; f \mid=2)+2 \bar{N}(r, b ; f \mid=3)+3 \bar{N}(r, b ; f \mid=4)+\cdots\} \\
& \quad \leq N\left(r, \frac{1}{L_{c}^{r} f-f}\right)+N\left(r, 0 ; f^{\prime} \mid f \neq 0\right) \\
& \quad \leq N\left(r, \frac{1}{L_{c}^{r} f-f}\right)+N\left(r, \frac{f^{\prime}}{f}\right) \\
& \quad \leq N\left(r, \frac{1}{L_{c}^{r} f-f}\right)+\bar{N}(r, f)+\bar{N}\left(r, \frac{1}{f}\right) .
\end{aligned}
$$

So (3.2) reduces to

$$
\bar{N}\left(r, \frac{1}{L_{c}^{r} f-a}\right)+\bar{N}\left(r, \frac{1}{L_{c}^{r} f-b}\right)
$$




$$
\begin{aligned}
& \leq N\left(r, \frac{1}{f-a}\right)+N\left(r, \frac{1}{f-b}\right) \\
& \leq T(r, f)+N\left(r, L_{c}^{r} f\right)+\bar{N}(r, f)+\bar{N}\left(r, \frac{1}{f}\right)+S(r, f) .
\end{aligned}
$$

Now from Lemma 2.5 we see that

$$
m\left(r, \frac{1}{f-a}\right)+m\left(r, \frac{1}{f-b}\right) \leq m\left(r, \frac{1}{L_{c}^{r} f}\right)+S(r, f),
$$

which, in view of (3.18) and the first fundamental theorem, yields

$$
T(r, f) \leq m\left(r, \frac{1}{L_{c}^{r} f}\right)+N\left(r, L_{c}^{r} f\right)+\bar{N}(r, f)+\bar{N}\left(r, \frac{1}{f}\right)+S(r, f) .
$$

Since

$$
\begin{aligned}
& N\left(r, \frac{1}{L_{c}^{r} f-a}\right)-\bar{N}\left(r, \frac{1}{L_{c}^{r} f-a}\right)+N\left(r, \frac{1}{L_{c}^{r} f-b}\right)-\bar{N}\left(r, \frac{1}{L_{c}^{r} f-b}\right) \\
& \quad \leq N\left(r, \frac{1}{\left(L_{c}^{r} f\right)^{\prime}}\right)+S(r, f),
\end{aligned}
$$

we get from (3.18) that

$$
\begin{aligned}
& N\left(r, \frac{1}{L_{c}^{r} f-a}\right)+N\left(r, \frac{1}{L_{c}^{r} f-b}\right) \\
& \quad \leq T(r, f)+N\left(r, L_{c}^{r} f\right)+N\left(r, \frac{1}{\left(L_{c}^{r} f\right)^{\prime}}\right)+\bar{N}(r, f)+\bar{N}\left(r, \frac{1}{f}\right)+S(r, f) .
\end{aligned}
$$

Again from (2.1) of Lemma 2.4 we notice that

$$
m\left(r, \frac{1}{L_{c}^{r} f}\right)+m\left(r, \frac{1}{L_{c}^{r} f-a}\right)+m\left(r, \frac{1}{L_{c}^{r} f-b}\right) \leq m\left(r, \frac{1}{\left(L_{c}^{r} f\right)^{\prime}}\right)+S(r, f) .
$$

So from (2.3), (3.21), and (3.22), using Lemmas 2.5 and 2.7 and applying the first fundamental theorem, we obtain

$$
\begin{aligned}
& m\left(r, \frac{1}{L_{c}^{r} f}\right)+2 T\left(r, L_{c}^{r} f\right)+O(1) \\
&= m\left(r, \frac{1}{L_{c}^{r} f}\right)+m\left(r, \frac{1}{L_{c}^{r} f-a}\right)+m\left(r, \frac{1}{L_{c}^{r} f-b}\right) \\
&+N\left(r, \frac{1}{L_{c}^{r} f-a}\right)+N\left(r, \frac{1}{L_{c}^{r} f-b}\right) \\
& \leq T\left(r, \frac{1}{\left(L_{c}^{r} f\right)^{\prime}}\right)+T(r, f)+N\left(r, L_{c}^{r} f\right)+\bar{N}(r, f)+\bar{N}\left(r, \frac{1}{f}\right)+S(r, f) \\
& \leq T(r, f)+m\left(r,\left(L_{c}^{r} f\right)^{\prime}\right)+N\left(r,\left(L_{c}^{r} f\right)^{\prime}\right)+N\left(r, L_{c}^{r} f\right)+\bar{N}(r, f)+\bar{N}\left(r, \frac{1}{f}\right)+S(r, f) \\
& \leq T(r, f)+m\left(r, \frac{\left(L_{c}^{r} f\right)^{\prime}}{L_{c}^{r} f}\right)+m\left(r, L_{c}^{r} f\right)+N\left(r,\left(L_{c}^{r} f\right)^{\prime}\right)+N\left(r, L_{c}^{r} f\right)+\bar{N}(r, f)
\end{aligned}
$$




$$
\begin{aligned}
& +\bar{N}\left(r, \frac{1}{f}\right)+S(r, f) \\
\leq & T(r, f)+T\left(r, L_{c}^{r} f\right)+N\left(r,\left(L_{c}^{r} f\right)^{\prime}\right)+\bar{N}(r, f)+\bar{N}\left(r, \frac{1}{f}\right)+S(r, f) .
\end{aligned}
$$

Thus, in view of (3.20), we get

$$
T\left(r, L_{c}^{r} f\right) \leq N\left(r, L_{c}^{r} f\right)+N\left(r,\left(L_{c}^{r} f\right)^{\prime}\right)+2 \bar{N}(r, f)+2 \bar{N}\left(r, \frac{1}{f}\right)+S(r, f) .
$$

Finally, using Lemmas 2.3 and 2.7 and (3.23) in (3.20), we get

$$
\begin{aligned}
T(r, f) & \leq m\left(r, \frac{1}{L_{c}^{r} f}\right)+N\left(r, L_{c}^{r} f\right)+\bar{N}(r, f)+\bar{N}\left(r, \frac{1}{f}\right)+S(r, f) \\
& \leq T\left(r, L_{c}^{r} f\right)+N\left(r, L_{c}^{r} f\right)+\bar{N}(r, f)+\bar{N}\left(r, \frac{1}{f}\right)+S(r, f) \\
& \leq 2 N\left(r, L_{c}^{r} f\right)+N\left(r,\left(L_{c}^{r} f\right)^{\prime}\right)+3 \bar{N}(r, f)+3 \bar{N}\left(r, \frac{1}{f}\right)+S(r, f) \\
& \leq 3 N\left(r, L_{c}^{r} f\right)+\bar{N}\left(r, L_{c}^{r} f\right)+3 \bar{N}(r, f)+3 \bar{N}\left(r, \frac{1}{f}\right)+S(r, f) \\
& \leq 3(k+1) N(r, f)+(k+4) \bar{N}(r, f)+3 \bar{N}\left(r, \frac{1}{f}\right)+S(r, f),
\end{aligned}
$$

which is a contradiction, and so $f \equiv L_{c}^{r} f$.

Proof of Theorem 1.4 Here we proceed with the same argument as in Theorem 1.3.

So (3.2) reduces to

$$
\begin{aligned}
& \bar{N}\left(r, \frac{1}{L_{c}^{r} f-a}\right)+\bar{N}\left(r, \frac{1}{L_{c}^{r} f-b}\right) \\
& \quad \leq N\left(r, \frac{1}{f-a}\right)+N\left(r, \frac{1}{f-b}\right) \leq T(r, f)+\bar{N}(r, f)+\bar{N}\left(r, \frac{1}{f}\right)+S(r, f) .
\end{aligned}
$$

Thus, in view of (3.19), using Lemma 2.5 and applying the first fundamental theorem, we get

$$
T(r, f) \leq m\left(r, \frac{1}{L_{c}^{r} f}\right)+\bar{N}(r, f)+\bar{N}\left(r, \frac{1}{f}\right)+S(r, f)
$$

Hence (3.21) reduces to

$$
\begin{aligned}
& N\left(r, \frac{1}{L_{c}^{r} f-a}\right)+N\left(r, \frac{1}{L_{c}^{r} f-b}\right) \\
& \quad \leq T(r, f)+N\left(r, \frac{1}{\left(L_{c}^{r} f\right)^{\prime}}\right)+\bar{N}(r, f)+\bar{N}\left(r, \frac{1}{f}\right)+S(r, f) .
\end{aligned}
$$

Hence (3.23) reduces to

$$
T\left(r, L_{c}^{r} f\right) \leq \bar{N}\left(r,\left(L_{c}^{r} f\right)^{\prime}\right)+2 \bar{N}(r, f)+2 \bar{N}\left(r, \frac{1}{f}\right)+S(r, f)
$$


Finally, by (3.25) and (3.27) we get

$$
T(r, f) \leq(k+1) N(r, f)+(k+4) \bar{N}(r, f)+3 \bar{N}\left(r, \frac{1}{f}\right)+S(r, f)
$$

which is a contradiction, and so $f \equiv L_{c}^{r} f$.

Proof of Theorem 1.5 Let $F_{1}=\left(\frac{f}{a}\right)^{n}, G_{1}=\left(\frac{\Delta_{c}^{k} f}{a}\right)^{n}$ and $F_{2}=\left(\frac{f}{b}\right)^{m}, G_{2}=\left(\frac{\Delta_{c}^{k} f}{b}\right)^{m}$. By the statement of the theorem it follows that $F_{i}$ and $G_{i}$ share $(1,2)$ for $i=1,2$.

First, suppose that (i) of Lemma 2.9 holds. Then we get

$$
T\left(r, F_{1}\right) \leq N_{2}\left(r, 0 ; F_{1}\right)+N_{2}\left(r, 0 ; G_{1}\right)+N_{2}\left(r, \infty ; F_{1}\right)+N_{2}\left(r, \infty ; G_{1}\right)+S\left(r, F_{1}\right)+S\left(r, G_{1}\right),
$$

that is,

$$
\begin{aligned}
n T(r, f) \leq & 2 \bar{N}\left(r, \frac{1}{f}\right)+2 \bar{N}\left(r, \frac{1}{\Delta_{c}^{k} f}\right)+2 \bar{N}(r, f)+2 \bar{N}\left(r, \Delta_{c}^{k} f\right) \\
& +S(r, f)+S\left(r, \Delta_{c}^{k} f\right) .
\end{aligned}
$$

In a similar way, we can obtain

$$
\begin{aligned}
n T\left(r, \Delta_{c}^{k} f\right) \leq & 2 \bar{N}\left(r, \frac{1}{f}\right)+2 \bar{N}\left(r, \frac{1}{\Delta_{c}^{k} f}\right)+2 \bar{N}(r, f)+2 \bar{N}\left(r, \Delta_{c}^{k} f\right) \\
& +S(r, f)+S\left(r, \Delta_{c}^{k} f\right) .
\end{aligned}
$$

Thus

$$
\begin{aligned}
& n\left\{T(r, f)+T\left(r, \Delta_{c}^{k} f\right)\right\} \\
& \quad \leq 4\left\{\bar{N}\left(r, \frac{1}{f}\right)+\bar{N}\left(r, \frac{1}{\Delta_{c}^{k} f}\right)+\bar{N}(r, f)+\bar{N}\left(r, \Delta_{c}^{k} f\right)\right\}+S(r, f)+S\left(r, \Delta_{c}^{k} f\right) \\
& \quad \leq 8\left\{T(r, f)+T\left(r, \Delta_{c}^{k} f\right)\right\}+S(r, f)+S\left(r, \Delta_{c}^{k} f\right),
\end{aligned}
$$

which is a contradiction for $n \geq 9$.

Next, considering cases (ii) and (iii) of Lemma 2.9, we get

$$
F_{1} \equiv G_{1} \text {, i.e., } f \equiv t_{1} \Delta_{c}^{k} f
$$

or

$$
F_{1} \cdot G_{1} \equiv 1, \quad \text { i.e., } f . \Delta_{c}^{k} f \equiv s_{1} \text {, }
$$

where $t_{1}^{n}=1$ and $s_{1}^{n}=a^{2 n}$.

Also,

$$
F_{2} \equiv G_{2} \text {, i.e., } f \equiv t_{2} \Delta_{c}^{k} f
$$


or

$$
F_{2} \cdot G_{2} \equiv 1, \quad \text { i.e., } f . \Delta_{c}^{k} f \equiv s_{2},
$$

where $t_{2}^{m}=1$ and $s_{2}^{m}=b^{2 m}$.

(1) Assume that (3.28) and (3.30) hold. Then $t_{1}=t_{2}$. Since $t_{1}^{n}=t_{2}^{m}=1$, where $n$ and $m$ are coprime, we get that $t_{1}=t_{2}=1$. It follows that $f \equiv \Delta_{c}^{k} f$.

(2) Assume that (3.28) and (3.31) hold. Then $t_{1}\left(\Delta_{c}^{k} f\right)^{2}=s_{2}$, which is impossible.

(3) Assume that (3.30) and (3.31) hold. Similarly to the argument as in (2), we obtain an impossible situation.

(4) Assume that (3.29) and (3.31) hold. Then $s_{1}=s_{2}$. Thus $a^{2 m n}=s_{1}^{m n}=s_{2}^{m n}=b^{2 m n}$, which is impossible.

Proof of Theorem 1.6 Let $F_{1}=\left(\frac{f}{a}\right)^{n}, G_{1}=\left(\frac{\Delta_{c}^{k} f}{a}\right)^{n}$ and $F_{2}=\left(\frac{f}{b}\right)^{m}, G_{2}=\left(\frac{\Delta_{c}^{k} f}{b}\right)^{m}$. By the statement of the theorem it follows that $F_{i}$ and $G_{i}$ share $(1,1)$ for $i=1,2$.

Let

$$
\left(\frac{F_{i}^{\prime \prime}}{F_{i}^{\prime}}-\frac{2 F_{i}^{\prime}}{F_{i}-1}\right)-\left(\frac{G_{i}^{\prime \prime}}{G_{i}^{\prime}}-\frac{2 G_{i}^{\prime}}{G_{i}-1}\right) \not \equiv 0 \text { for } i=1,2
$$

Now considering the functions $F_{1}$ and $G_{1}$, from Lemma 2.9 we get

$$
\begin{aligned}
T\left(r, F_{1}\right) \leq & N_{2}\left(r, 0 ; F_{1}\right)+N_{2}\left(r, 0 ; G_{1}\right)+N_{2}\left(r, \infty ; F_{1}\right)+N_{2}\left(r, \infty ; G_{1}\right)+\frac{1}{2} \bar{N}\left(r, 0 ; F_{1}\right) \\
& +\frac{1}{2} \bar{N}\left(r, \infty ; F_{1}\right)+S\left(r, F_{1}\right)+S\left(r, G_{1}\right) .
\end{aligned}
$$

Now as $f$ and $\Delta_{c}^{k} f$ are entire functions, we have

$$
n T(r, f) \leq 2 \bar{N}\left(r, \frac{1}{f}\right)+2 \bar{N}\left(r, \frac{1}{\Delta_{c}^{k} f}\right)+\frac{1}{2} \bar{N}\left(r, \frac{1}{f}\right)+S(r, f)+S\left(r, \Delta_{c}^{k} f\right) .
$$

In a similar way, we obtain

$$
n T\left(r, \Delta_{c}^{k} f\right) \leq 2 \bar{N}\left(r, \frac{1}{f}\right)+2 \bar{N}\left(r, \frac{1}{\Delta_{c}^{k} f}\right)+\frac{1}{2} \bar{N}\left(r, \frac{1}{\Delta_{c}^{k} f}\right)+S(r, f)+S\left(r, \Delta_{c}^{k} f\right) .
$$

Thus

$$
\begin{aligned}
n\{ & \left.T(r, f)+T\left(r, \Delta_{c}^{k} f\right)\right\} \\
\leq & 4\left\{\bar{N}\left(r, \frac{1}{f}\right)+\bar{N}\left(r, \frac{1}{\Delta_{c}^{k} f}\right)\right\}+\frac{1}{2}\left\{\bar{N}\left(r, \frac{1}{f}\right)+\bar{N}\left(r, \frac{1}{\Delta_{c}^{k} f}\right)\right\} \\
& +S(r, f)+S\left(r, \Delta_{c}^{k} f\right) \\
\leq & \frac{9}{2}\left\{T(r, f)+T\left(r, \Delta_{c}^{k} f\right)\right\}+S(r, f)+S\left(r, \Delta_{c}^{k} f\right),
\end{aligned}
$$

which is a contradiction for $n \geq 5$.

A similar contradiction holds for $m \geq 5$ while considering the functions $F_{2}$ and $G_{2}$. 
Next, suppose

$$
\left(\frac{F_{i}^{\prime \prime}}{F_{i}^{\prime}}-\frac{2 F_{i}^{\prime}}{F_{i}-1}\right)-\left(\frac{G_{i}^{\prime \prime}}{G_{i}^{\prime}}-\frac{2 G_{i}^{\prime}}{G_{i}-1}\right) \equiv 0 \quad \text { for } i=1,2
$$

Now considering the functions $F_{1}$ and $G_{1}$, by integration we get

$$
\frac{F_{1}^{\prime}}{\left(F_{1}-1\right)^{2}} \equiv A \frac{G_{1}^{\prime}}{\left(G_{1}-1\right)^{2}}
$$

where $A$ is a nonzero constant.

From this we obtain that $T\left(r, F_{1}\right)=T\left(r, G_{1}\right)+O(1)$, that is, $T\left(r, \Delta_{c}^{k} f\right)=T(r, f)+O(1)$.

Also, (3.32) implies that $F_{1}$ and $G_{1}$ share $(1, \infty)$.

Again, as $F_{1}$ and $G_{1}$ are entire functions, we can say that $F_{1}$ and $G_{1}$ share $(\infty, \infty)$ and

$$
N\left(r, \infty ; F_{1}\right)=N\left(r, \infty ; G_{1}\right)=0 .
$$

Now it is obvious that

$$
N_{2}\left(r, 0 ; F_{1}\right) \leq 2 N\left(r, \frac{1}{f}\right) \leq 2 T(r, f)+O(1) .
$$

Also, we obtain

$$
N\left(r, 0 ; G_{1}\right) \leq 2 T\left(r, \Delta_{c}^{k} f\right)+O(1) \leq 2 T(r, f)+S(r, f)
$$

So

$$
\limsup _{r \rightarrow \infty} \frac{N_{2}(r, 0 ; F)+N_{2}(r, 0 ; G)+2 \bar{N}(r, \infty ; F)}{T(r)} \leq \frac{4}{n}<1
$$

for $n \geq 5$. Thus using Lemma 2.8 , we get $F_{1}=G_{1}$ or $F_{1} \cdot G_{1}=1$.

Similarly, $F_{2}=G_{2}$ or $F_{2} \cdot G_{2}=1$.

Now if we take

$$
F_{1} \cdot G_{1} \equiv 1, \quad \text { i.e., } f^{n}\left(\Delta_{c}^{k} f\right)^{n} \equiv a^{2 n}
$$

then as $f$ and $\Delta_{c}^{k} f$ are both entire functions, we get $N\left(r, \infty ; \frac{\Delta_{c}^{k} f}{f}\right) \leq N\left(r, \frac{1}{f}\right)$.

Therefore

$$
\begin{aligned}
2 n T(r, f) & =2 T\left(r, F_{1}\right)+O(1) \leq T\left(r, \frac{1}{F_{1}^{2}}\right)+S(r, f) \\
& \leq T\left(r,\left(\frac{\Delta_{c}^{k} f}{f}\right)^{n}\right) \leq n T\left(r, \frac{\Delta_{c}^{k} f}{f}\right)+S(r, f) \leq n T(r, f)+S(r, f),
\end{aligned}
$$

which is a contradiction. So we have $F_{1} \equiv G_{1}$, that is, $f \equiv t_{1}\left(\Delta_{c}^{k} f\right)$, where $t_{1}^{n}=1$. Similarly, we have $f \equiv t_{2} \Delta_{c}^{k} f$, where $t_{2}^{m}=1$.

Thus in the same way as in Theorem 1.5 , we get $f \equiv \Delta_{c}^{k} f$. 
Proof of Theorem 1.7 Since $E_{f}\left(S_{1}, 1\right)=E_{\Delta_{c}^{k} f}\left(S_{1}, 1\right)$, in the line of the proof of Theorem 1.6, we can see that

$$
f \equiv t\left(\Delta_{c}^{k} f\right)
$$

where $t^{n}=1$.

Now if $b$ is a Picard value of $f$, by the assumption $E_{f}\left(S_{2}, 1\right)=E_{\Delta_{c}^{k} f}\left(S_{2}, 1\right)$ we know that $b$ is a Picard value of $\Delta_{c}^{k} f$. Again from (3.33) we see that $(t b)$ is a Picard value of $f$. Since $f$ is an entire function, we have $b=t b$. Thus $t=1$, and hence $f \equiv \Delta_{c}^{k} f$.

If $b$ is not a Picard value of $f$, then there exists $\alpha$ such that $f(\alpha)=\Delta_{c}^{k} f(\alpha)=b$. By (3.33) we obtain $b=t b$. Thus $t=1$, and hence $f \equiv \Delta_{c}^{k} f$.

Proof of Theorem 1.8 Since $E_{f}\left(S_{1}, 2\right)=E_{\Delta_{c}^{k} f}\left(S_{1}, 2\right)$, in the line of the proof of Theorem 1.5, we can see that

$$
f \equiv t\left(\Delta_{c}^{k} f\right)
$$

where $t^{n}=1$, or

$$
f \cdot \Delta_{c}^{k} f \equiv s,
$$

where $s^{n}=a^{2 n}$. Now we discuss the following cases.

Case 1.

Suppose $f$ and $\Delta_{c}^{k} f$ satisfy (3.34). We discuss the following subcases.

Subcase 1.1.

Assume that $b_{1}$ is not a Picard value of $f$. Then there exists $\alpha$ such that $f(\alpha)=b_{1}$. Since $E_{f}\left(S_{2}, 2\right)=E_{\Delta_{c}^{k} f}\left(S_{2}, 2\right)$, we obtain $\Delta_{c}^{k} f(\alpha)=b_{1}$ or $\Delta_{c}^{k} f(\alpha)=b_{2}$. If $\Delta_{c}^{k} f(\alpha)=b_{1}$, then by (3.34) we have $b_{1}=t b_{1}$. Thus $t=1$ and $f \equiv \Delta_{c}^{k} f$. If $\Delta_{c}^{k} f(\alpha)=b_{2}$, then by (3.34) we see that $b_{1}=t b_{2}$, which contradicts the assumption $b_{1}^{n} \neq b_{2}^{n}$.

Subcase 1.2.

Assume that $b_{2}$ is not a Picard value of $f$. Then in the similar way, we have $f \equiv \Delta_{c}^{k} f$.

Subcase 1.3.

Assume that $b_{1}$ and $b_{2}$ are Picard values of $f$. As $E_{f}\left(S_{1}, 2\right)=E_{\Delta_{c}^{k} f}\left(S_{1}, 2\right), b_{1}$ and $b_{2}$ are Picard values $f$ and $\Delta_{c}^{k} f$. Again by (3.34) we see that $\left(t b_{1}\right)$ and $\left(t b_{2}\right)$ are Picard values of $f$. Since a meromorphic function has at most two Picard values, $b_{1}=t b_{1}$ or $b_{1}=t b_{2}$. If $b_{1}=t b_{1}$, then $t=1$ and $f \equiv \Delta_{c}^{k} f$. If $b_{1}=t b_{2}$, then it contradicts the assumption $b_{1}^{n} \neq b_{2}^{n}$.

Case 2.

Suppose $f$ and $\Delta_{c}^{k} f$ satisfy (3.35). We discuss the following subcases.

Subcase 2.1.

Assume that $b_{1}$ is not a Picard value of $f$. Then there exists $\alpha$ such that $f(\alpha)=b_{1}$. Since $E_{f}\left(S_{2}, 2\right)=E_{\Delta_{c}^{k} f}\left(S_{2}, 2\right)$, we obtain that $\Delta_{c}^{k} f(\alpha)=b_{1}$ or $\Delta_{c}^{k} f(\alpha)=b_{2}$. If $\Delta_{c}^{k} f(\alpha)=b_{1}$, then by (3.35) we have $b_{1}^{2}=s$, which contradicts the assumption $b_{1}^{2 n}=a^{2 n}$. If $\Delta_{c}^{k} f(\alpha)=b_{2}$, then by (3.35) we have $\left(b_{1} b_{2}\right)=s$, which again contradicts the assumption $b_{1}^{n} b_{2}^{n} \neq a^{2 n}$.

\section{Subcase 2.2.}

Assume that $b_{2}$ is not a Picard value of $f$. Then in a similar way, we arrive at a contradiction. 


\section{Subcase 2.3.}

Assume that $b_{1}$ and $b_{2}$ are Picard values of $f$. Since $E_{f}\left(S_{2}, 2\right)=E_{\Delta_{c}^{k} f}\left(S_{2}, 2\right), b_{1}$ and $b_{2}$ are Picard values of $f$ and $\Delta_{c}^{k} f$. Again by (3.35) we see that $\frac{s}{b_{1}}$ and $\frac{s}{b_{2}}$ are Picard values of $f$. Since a meromorphic function has at most two Picard values, $b_{1}=\frac{s}{b_{1}}$ or $b_{1}=\frac{s}{b_{2}}$, and similarly in both situations, contradiction arises.

Proof of Theorem 1.9 The proof can be carried out in the line of Theorem 1.7.

Proof of Theorem 1.10 The proof can be carried out in the line of Theorem 1.8.

In this section, we have the following observation.

\section{Observation}

Considering $\alpha_{j}(\neq 1)$ for $j=1,2, \ldots, k$ as the roots of $\sum_{j=0}^{k}(-1)^{k-j} b_{j} z^{j}=1$, we claim that the general solution of the relation $L_{c}^{r} f \equiv f$ is of the form

$$
f(z)=\pi_{1}(z) \alpha_{1}^{\frac{z}{c}}+\pi_{2}(z) \alpha_{2}^{\frac{z}{c}}+\cdots+\pi_{k}(z) \alpha_{k}^{\frac{z}{c}}
$$

where $\pi_{i}(z+c)=\pi_{i}(z), 1 \leq i \leq k, c$ being a nonzero constant.

For $k=1$, we see that $L_{c}^{r} f=f$ implies $f(z+c)=\left(\frac{b_{0}+1}{b_{1}}\right) f(z)$. Clearly, in this case the general solution is

$$
f(z)=\pi_{1}(z)\left(\frac{b_{0}+1}{b_{1}}\right)^{\frac{z}{c}}=\pi_{1}(z) \alpha_{1}^{\frac{z}{c}},
$$

where $\alpha_{1}$ is a root of the equation $b_{1} z-b_{0}-1=0$.

Let us verify this fact:

$$
\begin{aligned}
L_{c}^{r} f & =b_{1} f(z+c)-b_{0} f(z) \\
& =b_{1}\left\{\pi_{1}(z+c) \alpha_{1}^{\frac{z+c}{c}}\right\}-b_{0}\left\{\pi_{1}(z) \alpha_{1}^{\frac{z}{c}}\right\} \\
& =\left(b_{1} \alpha_{1}-b_{0}\right) \pi_{1}(z) \alpha_{1}^{\frac{z}{c}} \\
& =\pi_{1}(z) \alpha_{1}^{\frac{z}{c}} \\
& =f(z) .
\end{aligned}
$$

For $k=2$, we see that $L_{c}^{r} f=f$ implies $b_{2} f(z+2 c)-b_{1} f(z+c)+b_{0} f(z)=f(z)$. Let $\alpha_{1}$ and $\alpha_{2}$ be the roots of the equation

$$
b_{2} z^{2}-b_{1} z+b_{0}-1=0
$$

In this case the general solution is of the form

$$
f(z)=\pi_{1}(z) \alpha_{1}^{\frac{z}{c}}+\pi_{2}(z) \alpha_{2}^{\frac{z}{c}} .
$$

Let us verify this fact:

$$
L_{c}^{r} f=b_{2} f(z+2 c)-b_{1} f(z+c)+b_{0} f(z)
$$




$$
\begin{aligned}
= & b_{2}\left\{\pi_{1}(z+2 c) \alpha_{1}^{\frac{z+2 c}{c}}+\pi_{2}(z+2 c) \alpha_{2}^{\frac{z+2 c}{c}}\right\}-b_{1}\left\{\pi_{1}(z+c) \alpha_{1}^{\frac{z+c}{c}}+\pi_{2}(z+c) \alpha_{2}^{\frac{z+c}{c}}\right\} \\
& +b_{0}\left\{\pi_{1}(z) \alpha_{1}^{\frac{z}{c}}+\pi_{2}(z) \alpha_{2}^{\frac{z}{c}}\right\} \\
= & \left(b_{2} \alpha_{1}^{2}-b_{1} \alpha_{1}+b_{0}\right) \pi_{1}(z) \alpha_{1}^{\frac{z}{c}}+\left(b_{2} \alpha_{0}^{2}-b_{1} \alpha_{0}+b_{0}\right) \pi_{2}(z) \alpha_{2}^{\frac{z}{c}} \\
= & \pi_{1}(z) \alpha_{1}^{\frac{z}{c}}+\pi_{2}(z) \alpha_{2}^{\frac{z}{c}} \\
= & f(z) .
\end{aligned}
$$

For $k=m$, we see that $L_{c}^{r} f=f$ implies $b_{m} f(z+m c)-b_{m-1} f(z+\overline{m-1} c)+\cdots+(-1)^{m} b_{0} f(z)=$ $f(z)$. Let $\alpha_{i}, 1 \leq \alpha_{i} \leq m$, be the roots of the equation

$$
b_{m} z^{m}-b_{m-1} z^{m-1}+\cdots+(-1)^{m} b_{0}-1=0 .
$$

In this case the general solution is of the form

$$
f(z)=\pi_{1}(z) \alpha_{1}^{\frac{z}{c}}+\pi_{2}(z) \alpha_{2}^{\frac{z}{c}}+\cdots+\pi_{m}(z) \alpha_{m}^{\frac{z}{c}}
$$

since

$$
\begin{aligned}
L_{c}^{r} f= & b_{m} f(z+m c)-b_{m-1} f(z+\overline{m-1} c)+\cdots+(-1)^{m} b_{0} f(z) \\
= & b_{m}\left\{\pi_{1}(z+m c) \alpha_{1}^{\frac{z+m c}{c}}+\cdots+\pi_{m}(z+m c) \alpha_{m}^{\frac{z+m c}{c}}\right\}-b_{m-1}\left\{\pi_{1}(z+\overline{m-1} c) \alpha_{1}^{\frac{z+\overline{m-1} c}{c}}\right. \\
& \left.+\cdots+\pi_{m}(z+\overline{m-1} c) \alpha_{m}^{\frac{z+\overline{m-1} c}{c}}\right\}+\cdots+(-1)^{m} b_{0}\left\{\pi_{1}(z) \alpha_{1}^{\frac{z}{c}}+\cdots+\pi_{m}(z) \alpha_{m}^{\frac{z}{c}}\right\} \\
= & \left\{b_{m} \alpha_{1}^{m}-b_{m-1} \alpha_{1}^{m-1}+\cdots+(-1)^{m} b_{0}\right\} \pi_{1}(z) \alpha_{1}^{\frac{z}{c}}+\cdots \\
& +\left\{b_{m} \alpha_{1}^{m}-b_{m-1} \alpha_{1}^{m-1}+\cdots+(-1)^{m} b_{0}\right\} \pi_{m}(z) \alpha_{m}^{\frac{z}{c}} \\
= & \left\{\pi_{1}(z) \alpha_{1}^{\frac{z}{c}}+\cdots+\pi_{m}(z) \alpha_{m}^{\frac{z}{c}}\right\} \\
= & f(z) .
\end{aligned}
$$

So we conjecture that the general solution of the equation $L_{c}^{r} f=f$ for any integer $k$ is

$$
f(z)=\pi_{1}(z) \alpha_{1}^{\frac{z}{c}}+\pi_{2}(z) \alpha_{2}^{\frac{z}{c}}+\cdots+\pi_{k}(z) \alpha_{k}^{\frac{z}{c}}
$$

where $\pi_{i}(z+c)=\pi_{i}(z), 1 \leq i \leq k$, where $c$ is a constant, and $\alpha_{j}(\neq 1), 1 \leq j \leq k$, are the roots of the equation $\sum_{j=0}^{k}(-1)^{k-j} b_{j} z^{j}=1$.

\section{An open question}

Due to suitable choice of the coefficients of the linear $c$-shift operator, Theorems 1.1 and 1.2 are valid for $L_{c}^{r} f$. So the following question is inevitable:

Do Theorems 1.1 and 1.2 hold if $L_{c} f$ is considered instead of $L_{c}^{r} f$ ? 
Funding

Neither of the authors has support of funding agencies.

Availability of data and materials

The data and materials used in the paper are properly cited.

Competing interests

The authors declare that they have no competing interests.

Authors' contributions

Both authors contributed equally to the writing of this paper. Both authors read and approved the final manuscript.

\section{Author details}

'Department of Mathematics, University of Kalyani, West Bengal, India. ²Department of Science and Humanities, Jangipur Government Polytechnic, West Bengal, India.

\section{Publisher's Note}

Springer Nature remains neutral with regard to jurisdictional claims in published maps and institutional affiliations.

Received: 20 August 2019 Accepted: 18 November 2019 Published online: 11 December 2019

\section{References}

1. Banerjee, A.: Meromorphic functions sharing one value. Int. J. Math. Math. Sci. 22, 3587-3598 (2005)

2. Banerjee, A.: Meromorphic functions sharing two sets. Czechoslov. Math. J. 57(132), 1199-1214 (2007)

3. Chen, Z.X., Yi, H.X.: On sharing values of meromorphic functions and their differences. Results Math. 63, 557-565 (2013)

4. Chiang, Y.M., Feng, S.J.: On the Nevanlinna characteristic $f(z+\eta)$ and difference equations in complex plane. Ramanujan J. 16, 105-129 (2008)

5. Halburd, R.G., Korhonen, R.: Finite order solutions and the discrete Painlevé equations. Proc. Lond. Math. Soc. 94 , 443-474 (2007)

6. Hayman, W.K.: Meromorphic Functions. The Clarendon Press, Oxford (1964)

7. Jiang, Y., Chen, Z.: Meromorphic functions share two values with its difference operator. An. Ştiinţ. Univ. 'Al.I. Cuza' laşi, Mat. 63(1), 169-175 (2017)

8. Lahiri, I.: Weighted value sharing and uniqueness of meromorphic functions. Complex Var. Theory Appl. 46, 241-253 (2001)

9. Lahiri, I.: Weighted sharing and uniqueness of meromorphic functions. Nagoya Math. J. 161, 193-206 (2001)

10. Lu, F., Lu, W.: Meromorphic functions sharing three values with their difference operators. Comput. Methods Funct. Theory 17(3), 395-403 (2017)

11. Mues, E., Steinmetz, N.: Meromorphe Funktionen die unit ihrer Ableitung Werte teilen. Manuscr. Math. 514(29), 195-206 (1979)

12. Rubel, L.A., Yang, C.C.: Values shared by an entire function and its derivative. In: Complex Anal, Proc. Conf., Univ. Kentucky, Lexington, Ky., 1976. Lecture Notes in Math., vol. 599, pp. 101-103. Springer, Berlin (1977)

13. Yi, H.X.: Meromorphic functions that share one or two values. Complex Var. Theory Appl. 28, 1-11 (1995)

14. Yi, H.X., Yang, C.C.: Uniqueness Theory of Meromorphic Functions. Science Press, Beijing (1995)

15. Zhang, J., Liao, L.W.: Entire functions sharing some values with their difference operators. Sci. China Math. 57 , 2143-2152 (2014)

\section{Submit your manuscript to a SpringerOpen ${ }^{\circ}$ journal and benefit from:}

- Convenient online submission

- Rigorous peer review

- Open access: articles freely available online

- High visibility within the field

Retaining the copyright to your article 